\title{
Écologisation : entre sciences, conventions et pratiques
}

La notion d'écologisation désigne les processus par lesquels l'environnement est pris en compte dans les politiques publiques, dans les organisations, voire dans les pratiques professionnelles. L'environnement est ici entendu largement, il comprend aussi bien les questions sanitaires que les milieux naturels ou anthropisés qui supportent la vie. Devenue presque une notion de sens commun, l'écologisation peut aussi être théorisée: il s'agit alors de construire des schémas de compréhension et d'explication des processus de changements, bref de décrire et caractériser ces processus ${ }^{1}$. Ces transformations mêlent inextricablement des bifurcations techniques, des réformes normatives et des mutations économiques, ce qui rend le changement peu prévisible dans ses issues concrètes.

La théorie de la modernisation écologique et son avatar, celle de la transition écologique, occupent une place dominante dans ces efforts de théorisation. Ces théories tirent leur force du modèle qu'elles proposent : ce sont les efforts combinés des innovateurs, des associations et du marché qui, par différents processus, produisent peu à peu les changements en direction d'une société écologique, d'une économie propre. Dans ce modèle, où la complexité est de règle, l'État n'est plus un acteur central, l'innovation vient des acteurs civiques ou industriels et des sciences et de leurs interactions à différentes échelles. L'État joue au mieux un rôle de coordinateur. La modernisation écologique constitue en fait le référentiel contemporain des politiques d'environnement, réalisant un compromis savant entre la vision néolibérale et les critiques environnementales. Elle inspire d'ailleurs explicitement les politiques européennes d'environnement.

Cette perspective entretient un certain rapport avec les disciplines. $\mathrm{Ou}$, plus exactement, elle appelle au développement massif d'un type d'activité scientifique. Plutôt que de mobiliser les vieux couples (fondamental/

1 Le lecteur trouvera dans ce numéro un « dossier » constitué de plusieurs textes issus du colloque «Écologisation des politiques et des pratiques agricoles » qui s'est tenu à proximité d'Avignon en 2011. appliqué, etc.), il est pertinent peut-être de distinguer deux types d'activité. D'un côté, les recherches qui s'attachent à définir des objets nouveaux (biologiques, mathématiques, physiques); c'est l'activité qui, organisée en disciplines, appuyée sur des méthodes, fait advenir du nouveau. Ces objets ne sont pas ceux sur lesquels portent les décisions environnementales. D'un autre côté, existe une autre activité scientifique que nous appellerons, faute de mieux, de production de conventions : il s'agit ici, par différentes médiations, de relier des connaissances scientifiques à des pratiques sociales, à des normes, à des politiques publiques. Les normes de toxicité, les services écosystémiques, les évaluations d'impact - la liste pourrait s'allonger indéfiniment-, sont autant de construits qui articulent des savoirs scientifiques issus des disciplines et des univers sociaux. On peut définir de la même manière les innovations techniques qui sont aussi des articulations de savoirs, de savoir-faire et de normes. Dans la mesure où elles revendiquent d'écologiser le monde, on peut les appeler conventions écologiques.

La production de ces conventions occupe aujourd'hui une part importante, voire majeure, des activités dans le monde de la recherche et elles peuvent être décomposées en multiples travaux: collectes de données, bases de données, cartographies, instruments d'évaluation, etc. La définition de listes d'espèces menacées ou celle des produits toxiques appartiennent à cette catégorie des conventions environnementales qui associent savoirs et normes.

L'écologisation a besoin de s'appuyer sur de telles conventions pour se déployer. C'est à travers elles que le mouvement d'écologisation se concrétise, se précise et s'oriente. On ne peut pas imputer aux seuls scientifiques la production de ces conventions, car précisément elles résultent d'interactions multiples qui impliquent, à la fois, engagement des chercheurs et maintien d'une autonomie des disciplines. Elles mettent en relation des pratiques de recherche et des pratiques sociales dans des rapports compliqués qui restent souvent cachés dans les instruments qui en résultent. La capacité à produire de 
telles conventions fait l'objet de multiples attentes mais, en même temps, suscite quelques craintes.

Une première crainte est une monopolisation de ce travail de convention par des "experts », qu'ils soient scientifiques ou associatifs, que ce soit au nom de la compétence scientifique ou au nom d'une légitimité $\mathrm{d}^{\prime}$ action. Des convergences entre experts et ONG peuvent d'ailleurs s'observer dans le domaine de la conservation, de la même façon qu'experts et industriels se retrouvent autour des innovations techniques.

Une seconde crainte prolonge la première car la monopolisation risque aussi de se traduire par l'imposition de points de vue particuliers qui viendraient alors coloniser (et normaliser) les pratiques sociales. C'est que la production de telles conventions suppose des choix qui sont souvent cachés dans les multiples opérations qui y conduisent.

Symétriquement, un certain nombre de scientifiques peuvent craindre l'absorption ou la dilution de leurs pratiques de recherche dans des préoccupations d'expertise qui viennent les détourner ou les instrumentaliser et, à terme, détruire ce qui fait l'aventure propre de leur discipline.

Entre, d'une part, la nécessité évidente de telles conventions entre recherche et pratiques ou politiques et, d'autre part, ces risques, il est nécessaire d'affirmer que nul point de vue supérieur ou englobant ne peut prétendre s'imposer à tous les autres. Le pluralisme des points de vue n'implique pas un relativisme qui réduirait toutes ces pratiques à n'être que des stratégies. Il se justifie par la diversité irréductible du réel et la pluralité des manières de le révéler.

Le dialogue des disciplines est une tâche ardue et difficile. Il est sans aucun doute ce que NSS, depuis ses origines, a tâché de favoriser. Mais ce dialogue n'est sans doute porteur de potentialités que s'il s'élargit à un dialogue avec les autres pratiques sociales que concernent les objets sur lesquels ces conventions se construisent. De ce point de vue, il faut sans doute considérer les pratiques scientifiques comme des pratiques comme les autres.

La modernisation écologique n'est certes pas la seule théorisation possible du changement. Mais c'est cette théorie - avec ses multiples variantes - qui sert aujourd'hui de référentiel à la plupart des actions et des recherches qui se préoccupent d'écologiser notre monde. Son devenir et sa transformation, son infléchissement ou sa radicalisation vont dépendre de la manière dont nous serons capables de réellement faire dialoguer les pratiques dans la formulation des conventions écologiques.

Marc Mormont 